\title{
Doença celíaca: repercussões do diagnóstico tardio
}

\author{
Celiac disease: repercussions of late diagnosis \\ Enfermedad celíaca: repercusiones del diagnóstico tardío
}

Isabela Bianca Rodrigues Fernandes ${ }^{1}$, Carla Moreira da Costa ${ }^{1}$, Cecília Faria de Oliveira ${ }^{1}$, Fernando Augusto Maciel Soares ${ }^{1}$, Gustavo Marques Miranda ${ }^{1}$, Lucas Gabriel de Moraes Silva ${ }^{1}$, Lucas Gomes Teixeira de Sousa ${ }^{1}$, Marvin Michelon de Oliveira ${ }^{1}$, Raquel Araújo Lucas Novacki ${ }^{1}$, Danielle Soares Gardone $^{2 *}$.

\section{RESUMO}

Objetivo: Discutir, a partir da revisão da literatura atual sobre o tema, as recomendações para o diagnóstico da Doença Celíaca (DC), bem como seus principais dificultadores e as consequências associadas ao diagnóstico tardio desta enteropatia. Revisão bibliográfica: Pesquisas epidemiológicas recentes têm mostrado que a DC pode acometer indivíduos de todo o mundo, de diferentes gêneros, etnias e idades, refutando a crença de ser uma doença característica da infância, como era postulado anteriormente. Entretanto, sua ampla variedade clínica, com ausência de manifestações exclusivas à essa patologia, dificultam sua identificação precoce. Dessa forma, os estudos mostram que o diagnóstico tardio associa-se a uma vasta gama de complicações, que prejudicam o bem estar e a qualidade de vida dos pacientes. Considerações finais: É de suma importância a capacitação e atualização dos profissionais da saúde sobre a sintomatologia e métodos diagnósticos da DC, de forma a identificar e tratar essa patologia em um menor tempo, minimizando, assim, as comorbidades associadas ao diagnóstico tardio.

Palavras-chave: Doença celíaca, Diagnóstico tardio, Qualidade de vida, Dieta livre de glúten.

\begin{abstract}
Objective: To discuss, based on a review of the current literature on the subject, the recommendations for the diagnosis of Celiac Disease (CD), as well as its main obstacles and consequences associated with late diagnosis of this enteropathy. Literature review: Recent epidemiological research has shown that CD can affect individuals all over the world, of different genders, ethnicities and ages, refuting the belief that it is a characteristic disease of childhood, as previously postulated. However, its wide clinical variety, with the absence of manifestations exclusive to this pathology, make its early identification difficult. Thus, studies show that late diagnosis is associated with a wide range of complications, which affect the well-being and quality of life of patients. Final considerations: It is extremely important to train and update health professionals on the symptoms and diagnostic methods of $C D$, in order to identify and treat this pathology in a shorter time, thus minimizing the comorbidities associated with late diagnosis
\end{abstract}

Key words: Celiac disease, Delayed diagnosis, Quality of life, Diet gluten-free.

\section{RESUMEN}

Objetivo: Discutir, a partir de una revisión de la literatura actual sobre el tema, las recomendaciones para el diagnóstico de la Enfermedad Celíaca (EC), así como sus principales obstáculos y las consecuencias

\footnotetext{
${ }^{1}$ Faculdade Morgana Potrich (FAMP), Mineiros - GO.

*E-mail: dani.gardone@gmail.com

2 Universidade Federal de Viçosa (UFV), Viçosa - MG.
} 
asociadas al diagnóstico tardío de esta enteropatía. Revisión de la literatura: Investigaciones epidemiológicas recientes han demostrado que la EC puede afectar a personas de todo el mundo, de diferentes géneros, etnias y edades, refutando la creencia de que es una enfermedad característica de la infancia, como se postuló anteriormente. Sin embargo, su amplia variedad clínica, con la ausencia de manifestaciones exclusivas de esta patología, dificulta su identificación precoz. Así, los estudios muestran que el diagnóstico tardío se asocia a una amplia gama de complicaciones, que afectan el bienestar y la calidad de vida de los pacientes. Consideraciones finales: Es de suma importancia capacitar y actualizar a los profesionales de la salud sobre los síntomas y métodos diagnósticos de la EC, con el fin de identificar y tratar esta patología en un menor tiempo, minimizando así las comorbilidades asociadas al diagnóstico tardío.

Palabras clave: Enfermedad celíaca, Diagnóstico tardío, Calidad de vida, Dieta sin gluten.

\section{INTRODUÇÃO}

A Doença Celíaca (DC) é uma manifestação crônica e autoimune que envolve um processo inflamatório com alterações da permeabilidade intestinal e da resposta do organismo quando este é exposto ao glúten, que é um complexo protéico encontrado em alimentos como trigo, centeio, aveia e cevada. Esta patologia acomete pessoas geneticamente predispostas, embora fatores imunológicos e ambientais também possam influenciar no surgimento da doença (BESSA CC, et al., 2020).

O processo inflamatório que ocorre na mucosa intestinal é caracterizado pela degradação e atrofia das vilosidades, o que leva à má absorção e desencadeia uma série de sintomas gastrointestinais e extraintestinais. As manifestações clínicas gastrointestinais mais comuns, incluem diarreia, dor abdominal, constipação, flatulência, esteatorreia e distensão abdominal. Já entre os sintomas extra-intestinais, têm-se anemia, deficiência de vitaminas e alterações do estado psicológico (MURPHY AK, et al., 2020).

A DC é considerada uma das alterações do trato digestivo mais comuns. A prevalência é de aproximadamente $1 \%$ da população mundial e possui uma alta variação de distribuição entre os países, mostrando-se fortemente influenciada pela genética. No Brasil, as regiões que possuem a maior prevalência são a Sul e Sudeste, influenciada pela forte colonização europeia, mas também pela maior disponibilidade e acesso aos métodos diagnósticos comparado às outras regiões brasileiras (KARHUS LL, et al., 2021).

A DC é uma patologia que acomete todas as idades, porém tem maior prevalência em crianças de seis meses a cinco anos. Também foi observada maior prevalência em mulheres, sendo a proporção de duas mulheres para cada um homem diagnosticado. Entretanto, esse índice vem aumentando e estudos mostraram que a DC é mais frequente do que se imaginava. Estima-se que grande parte dos pacientes são subdiagnosticados, seja por dificuldade de acesso aos métodos diagnósticos ou por falta de conhecimento por parte da população e dos profissionais de saúde (BRASIL, 2015; GREEN PH, et al., 2015).

O diagnóstico da doença, embora seja de difícil estabelecimento, visto que não há sinais e sintomas isolados pertinentes unicamente à $\mathrm{DC}$, deve ser cogitado a partir de sintomas como flatulências, diarreia crônica e distensão abdominal, e também, de achados laboratoriais como deficiência de ácido fólico, hipocalemia e elevação de transaminases. O diagnóstico feito tardiamente traz consequências como anemia, deficiência de nutrientes, depressão e câncer, logo, faz se necessária a detecção precoce do problema por meio de exames e características histopatológicas sugestivas, a fim de evitar maior estresse a um corpo que já está debilitado (SANTOS AS e RIBEIRO CG, 2019).

Atualmente o único tratamento disponível é a Dieta Isenta de Glúten (DIG) que, quando bem implementada, causa a cessação dos sintomas clínicos, melhora os quadros de deficiências nutricionais e reduz o risco de desenvolver doenças associadas à DC (SOOD A, et al., 2014).

Diante desse contexto, o objetivo do presente trabalho é discutir, a partir da revisão da literatura atual sobre o tema, as recomendações para o diagnóstico da doença celíaca, bem como seus principais dificultadores e as consequências associadas ao diagnóstico tardio desta enteropatia. 


\section{REVISÃO BIBLIOGRÁFICA}

A DC é um acometimento clínico com imunidade e resistência ao glúten que sensibiliza indivíduos com predisposição genética, causando uma inflamação crônica no intestino delgado (RODRIGO L, 2019). Assim, o sistema imune inato e adaptativo é ativado após a ingestão de peptídeos de glúten contidos em alimentos como cevada, centeio e trigo (TAN IL, et al., 2020).

Para que a doença se desenvolva, é necessário que subtipos do antígeno leucocitário humano HLA-DQ2 e HLA-DQ8 estejam presentes, pois são eles que expõem os peptídeos do glúten ao sistema imunológico. Entretanto, embora até $40 \%$ da população seja portadora do genótipo HLA-DQ2 ou HLA-DQ8, apenas 2\% a $3 \%$ dos portadores desenvolvem a DC (LEONARD MM, et al., 2017).

Tem-se observado um aumento na incidência da DC nas últimas duas décadas, sendo este um acometimento clínico global. Esse aumento pode estar relacionado à melhoria dos métodos diagnósticos da DC, ao aumento real do distúrbio de base imunológica e, também, pela associação de fatores ambientais que podem estimular a perda de tolerância ao glúten (REJ A e SANDERS DS, 2021; LEBWOHL B e RUBIOTAPIA A, 2020).

Inicialmente, essa patologia era considerada uma condição gastrointestinal relativamente rara e que afetava quase exclusivamente crianças brancas. Entretanto, dados epidemiológicos atuais revelam que a DC é uma comumente encontrada em todo o mundo, afetando todas as idades, até mesmo os idosos, e quase todas as raças. Estima-se que $1 \%$ da população mundial seja acometida por esta patologia, porém observase grande variação regional, bem como uma incidência crescente, atribuída a uma sinergia de fatores genéticos e ambientais (KARHUS LL, et al., 2021; LEONARD MM, et al., 2017; BATHRELLOU E, et al., 2018).

Existem três formas principais de apresentação clínica da DC: a forma clássica ou típica, a não clássica ou atípica, e a assintomática ou silenciosa (BRASIL, 2015). A forma clássica caracteriza-se por quadros de diarreia, distensão abdominal e perda ponderal. Entretanto, outras manifestações como dispepsia, flatulência, esteatorreia, constipação, anorexia, vômitos e refluxo podem prevalecer em até $4 \%$ dos pacientes, desmistificando a tríade clássica (MURPHY AK, et al., 2020; ESMAEILZADEH A, et al., 2016).

A forma não clássica, por sua vez, caracteriza-se por poucos sintomas, podendo ser assintomática, em que as manifestações digestivas clássicas estão ausentes ou, quando presentes, ocupam um segundo plano. Outros achados associados ao quadro não clássico estão relacionados à ocorrência de deficiências nutricionais e traços neuropsiquiátricos com cefaleia, ataxia, irritabilidade, ansiedade, depressão e distúrbios osteoarticulares e musculares como artrite, osteoporose, osteomalácia, cãibras e miopatia (ESMAEILZADEH A, et al., 2016).

Já a forma assintomática (silenciosa) retrata uma alteração sorológica e histológica da mucosa intestinal, semelhante a DC, porém sem manifestações clínicas. Existe, ainda, a dermatite herpetiforme que é uma manifestação dermatológica da DC e se apresenta com lesões cutâneas do tipo bolhoso e intensamente pruriginoso (MAISIAK E, et al., 2021; MURPHY AK, et al., 2020; ESMAEILZADEH A, et al., 2016).

De acordo como Protocolo Clínico e Diretrizes Terapêuticas da Doença Celíaca proposto pelo Ministério da Saúde em 2015, a realização de endoscopia digestiva alta com biópsia de intestino delgado associado ao exame histopatológico, é considerado o padrão-ouro de diagnóstico. Os marcadores sorológicos como o anticorpo antiendomísio, o anticorpo antitransglutaminase e anticorpo antigliadina, por sua vez, são úteis na triagem indivíduos que deverão ser submetidos à biópsia de intestino delgado (BRASIL, 2015).

Os anticorpos antitransglutaminase tecidual IgA e o antiendomísio IgA são específicos para DC e têm sensibilidade e especificidade que podem variar de $90 \%$ a $95 \%$ em adultos e crianças maiores de oito anos. Já o uso da antigliadina vem sendo descontinuado ao longo dos anos, por possuir menor sensibilidade e especificidade, principalmente em adultos. Deve-se considerar, ainda, em indivíduos que apresentam deficiência de imunoglobulina A IgA podem ocorrer resultados falsos negativos dos testes sorológicos antiendomísio e antitransglutaminase da classe IgA. Por este motivo, indica-se como testes diagnósticos iniciais da DC a dosagem sérica simultânea destes anticorpos da classe $\lg \mathrm{A}$ e da imunoglobulina $\mathrm{A}$ e, caso 
haja IgA baixa, deve-se testar os mesmos anticorpos da classe IgG (BRASIL, 2015; RODRIGO L, 2020; LIMA CSA, et al., 2021).

Como método complementar, o teste de HLA pode ser útil quando há discrepância entre os estudos sorológicos e os achados histológicos, para avaliar melhor se a doença celíaca é possível, uma vez que $95 \%$ dos portadores de DC são HLA DQ2 positivos e o restante é HLA DQ8 positivo (BATHRELLOU E, et al., 2018; LEONARD MM, et al., 2017).

Entretanto, mesmo com protocolo bem estabelecido, o diagnóstico da DC ainda é difícil e demorado, uma vez que não há manifestações clínicas exclusivas a essa patologia. Soma-se a isso o despreparo de muitos profissionais de saúde a respeito dos métodos adequados, o acesso limitado a especialistas, a apresentação atípica da doença e, como resultado, têm-se um atraso no diagnóstico que pode chegar a 12 anos (CICHEWICZ AB, et al., 2019; RODRIGO L, 2019). Fuchs V, et al. (2018) constataram que um atraso superior a três anos no diagnóstico da doença celíaca está associado à redução do bem-estar dos pacientes, bem como relaciona-se ao aumento do uso de medicamentos e maior necessidade de cuidados de médicos no geral.

Em adultos, ao contrário das formas infantis clássicas, é menos frequente os casos diagnosticados a partir de manifestações intestinais. Nesses casos, predominam os sintomas atípicos e quadros como a anemia por deficiência de ferro, deficiência de folato e vitamina B12, osteoporose, hipoplasia do esmalte dentário, artralgias ou artrites, atraso puberal, irregularidade do ciclo menstrual, esterilidade e abortos de repetição. Muitos desses quadros apresentam melhora importante após o diagnóstico e adesão à DIG, porém, outros podem gerar sequelas que perdurarão por toda a vida (MURPHY AK, et al., 2020; ESMAEILZADEH A, et al., 2016; LIMA CSA, et al., 2021).

Desta forma, muitos pacientes celíacos apresentam deficiências nutricionais ao diagnóstico, atribuídas principalmente à histopatologia da doença, entretanto, após o início da GID, uma melhora geral nos índices do estado nutricional é observada. A anemia ferropriva, por exemplo, é observada em mais de $30 \%$ dos adultos no momento do diagnóstico, sendo essa a segunda apresentação clínica mais comum após a diarreia. Assim, pacientes com anemia ferropriva sem outras causas, e que não respondem à ingestão oral de terapia com ferro, devem ser testados para DC (GREEN PH, et al., 2015).

Já a osteoporose pode estar presente em $10 \%$ dos pacientes no diagnóstico de DC, visto que a baixa densidade mineral óssea (DMO) é um achado comum entre pacientes com a doença. No entanto, a frequência com que os pacientes apresentam osteoporose é provavelmente subnotificada, uma vez que a maioria dos casos não será aparente até que ocorra uma complicação clínica, como uma fratura espontânea (DI STEFANO M, 2013; LEONARD MM, et al., 2017).

A infertilidade é outro importante achado e estudos já comprovaram uma associação significativa entre mulheres com diagnóstico de infertilidade e doença celíaca não diagnosticada. A suspeita é que a presença dos anticorpos antitransglutaminase interligados às células endometriais não permite a implantação do trofoblasto no sítio endometrial, sucedendo na apoptose do mesmo. Portanto, a resposta imunológica derivada do glúten nos organismos dessas mulheres acarreta infertilidade e até mesmo abortos de repetição. Dessa forma, mulheres com infertilidade com etiologia desconhecida devem ser examinadas para DC, uma vez que estas podem ter probabilidade até seis vezes maior de possuir esta patologia (LASA JS, et al., 2014; SINGH P, et al., 2016).

Quadros neurológicos e manifestações neuropsiquiátricas são manifestações frequentes, podendo acometer até $22 \%$ dos pacientes com DC. Dentre essas manifestações, observa-se a neuropatia periférica, a epilepsia, a ansiedade e a depressão. Apesar da etiologia poder ser atribuída a deficiências nutricionais, como a de vitamina B12, o papel da dieta no manejo dessas condições ainda é conflitante na literatura e inflamação crônica e mecanismos de base imunológica também são citados como causas possíveis. Deve-se considerar também que preocupações constantes com as escolhas alimentares e dificuldades de socialização associadas à DIG podem representar um fardo psicológico e serem associadas a quadros de ansiedade e depressão (THAWANI SP, et al.; 2015; ZINGONE F, et al., 2015). 
As doenças malignas também são consideradas uma morbidade comum em pacientes celíacos, apesar de não haver consenso na literatura sobre a associação da DC com essas patologias. Entretanto, um risco aumentado de desenvolver doenças linfoproliferativas e especialmente o linfoma não Hodgkin, é bem documentado por estudos, sendo que este aumento pode variar de três a seis vezes quando comparado a população geral (BATHRELLOU E, et al., 2018; LESLIE LA, et al., 2012).

Karhus LL, et al. (2020), ao analisarem estudos com total de 16.776 indivíduos, observaram a associação entre o aumento do risco de câncer em geral, doenças cardiovasculares e a DC não diagnosticada. Os principais tipos de cânceres encontrados foram o câncer de cabeça e pescoço, câncer de mama, câncer de útero e câncer gastrointestinal. Ainda assim, os autores não obtiveram dados suficientes para vincular 0 diagnóstico tardio dessa enteropatia com a maior mortalidade dos indivíduos.

Entretanto, muitos estudos relatam aumento da taxa de mortalidade em pacientes com DC em comparação com a população em geral, sendo esse risco aumentado em cerca de $40 \%$ para morte por todas as causas, porém com redução dessas taxas à medida que aumenta o tempo de diagnóstico. Ainda assim, esse risco pode perdurar por anos, principalmente naqueles diagnosticados na idade adulta e após muitos anos de início dos sintomas. Os estudos avaliam ainda que, quando avaliada a mortalidade por causa específica, a causa mais comum de morte entre pacientes com DC são neoplasias malignas, doenças vasculares e, em menor proporção, doenças respiratórias (BATHRELLOU E, et al., 2018; LEONARD MM, et al., 2017).

O único tratamento efetivo para doença celíaca é a DIG, ou seja, a exclusão dos alimentos que tenham em sua composição ingredientes como trigo, centeio e cevada. Com o início do tratamento é observada a regressão dos sintomas e a melhora histológica da mucosa intestinal, entretanto, na vigência de diagnóstico tardio ou a não aderência ao tratamento podem acarretar em alterações na absorção de diversos nutrientes por um período de tempo prolongado, podendo resultar em quadros de hipersensibilidade (BRASIL, 2015).

Sabe-se, entretanto, que a principal dificuldade para o manejo e controle da DC é a adesão ao tratamento de forma efetiva. Diversas barreiras como o acesso às informações de qualidade, educação alimentar, acompanhamento nutricional, necessidade de alimentação em restaurantes ou em outros ambientes, contaminação cruzada e o alto custo dos alimentos sem glúten, são fatores que tornam as mudanças de hábitos um desafio a ser superado pelos pacientes e médicos (BESSA CC, et al., 2019). Porém, a importância da adesão ao tratamento precisa ser constantemente reforçada visto que, mesmo com alto nível de adesão à dieta proposta, a ingestão acidental de glúten ou à contaminação de alimentos considerados seguros resultam em sintomas refratários e histologia persistentemente anormal na DC (ROSTAMI K, et al., 2017).

Tan IL, et al. (2020) descreveram que, quando o diagnóstico é realizado de forma tardia, mesmo após a introdução da DIG, a resolução de sintomas ocorre de forma lenta. Logo, o diagnóstico precoce, assim como o início da dieta sem glúten, tem impacto direto na qualidade de vida, abordando desde a dor, desconforto e redução do risco de comorbidades até sintomas relacionados à ansiedade e depressão (MAISIAK E, et.al., 2021).

Alguns estudos mostram, ainda, que em alguns casos os celíacos podem ser não responsivos ou refratários ao tratamento com a DIG. Essas duas condições são diferentes e nos casos não responsivos há a permanência e recorrência dos sintomas em pacientes com DIG há mais de 12 meses. Nessa situação, as causas mais comuns são a contaminação cruzada, síndrome do intestino irritável, supercrescimento bacteriano do intestino delgado, colite microscópica, intolerância à lactose e outras alergias alimentares (PENNY HA, et al., 2020).

Já a DC Refratária (DCR), mais rara, se caracteriza pela permanência da atrofia intestinal com sintomas de má absorção por período superior a 12 meses após incorporação da dieta. A DCR pode ser primária (DCRI) ou secundária (DCRII) e essa divisão se baseia na presença ou não de células inflamatórias. Nesses casos, pode haver a necessidade de tratamento farmacológico com corticosteroides sistêmicos, quimioterápicos, imunossupressores e anti-inflamatórios não esteroidais (CICHEWICZ AB, et al., 2019).

Assim, considerando todo o quadro clínico que pode relacionar-se à DC, os protocolos recomendam que o monitoramento dos pacientes deve ocorrer anualmente com avaliação recorrente quanto a adesão ao 
tratamento, a necessidade de acompanhamento nutricional especializado e, se necessário, realização exames de hematológicos específicos e reavaliação quanto às comorbidades associadas (NATIONAL INSTITUTE FOR HEALTH AND CARE EXCELLENCE, 2015).

\section{CONSIDERAÇÕES FINAIS}

A partir da pesquisa bibliográfica realizada, constatou-se que a Doença Celíaca possui prevalência crescente, podendo atingir indivíduos de qualquer etnia, região e idade, não somente na infância como acreditava-se no passado. Porém, as falhas em realizar um diagnóstico preciso a partir do início dos sintomas estão associadas ao desenvolvimento de comorbidades que podem comprometer significativamente a qualidade de vida desses indivíduos. Portanto, reforça-se a importância de que os profissionais de saúde estejam atualizados tanto sobre a variável gama sintomatológica da DC e seus métodos diagnósticos, quanto sobre o correto acompanhamento desses pacientes a fim de reduzir as consequências que surgem a partir do reconhecimento tardio, bem como da não adesão ao tratamento adequado.

\section{REFERÊNCIAS}

1. BATHRELLOU E, et al. Celiac disease and non-celiac gluten or wheat sensitivity and health in later life: A review. Maturitas, 2018;112:29-33.

2. BESSA CC, et al. Health control for celiac patients: an analysis according to the Pender Health Promotion model, Texto \& contexto enfermagem, 2020, 29: e2080420.

3. BRASIL. Ministério da Saúde. Portaria № $1149.2015 . \quad$ Disponível em: https://portalarquivos2.saude.gov.br/images/pdf/2015/novembro/13/Portaria-SAS-MS---1149-de-11-de-novembro-de2015.pdf. Acessado em: 12 de junho de 2021.

4. CICHEWICZ AB, et al. Diagnosis and Treatment Patterns in Celiac Disease. Digestive diseases and sciences, 2019; 64: 2095-2106.

5. DI STEFANO M, et al. Bone mass and mineral metabolism alterations in adult celiac disease: pathophysiology and clinical approach. Nutrients, 2013; 5:4786-4799.

6. ESMAEILZADEH A, et al. Adult Celiac Disease: Patients Are Shorter Compared with Their Peers in the General Population. Middle East Journal of Digestive Diseases, 2016;8(4):303-309.

7. FUCHS V, et al. Delayed celiac disease diagnosis predisposes to reduced quality of life and incremental use of health care services and medicines: A prospective nationwide study. United European gastroenteroly jornal, 2018;6(4):567-575.

8. GREEN PH, et al. Clinical manifestations of celiac disease. Digestive Diseases, 2015;33(2):137-140.

9. KARHUS LL, et al. Symptoms and biomarkers associated with undiagnosed celiac seropositivity. BMC Gastroenteroly, 2021;21(1):90.

10. LASA JS, et al. Risk of infertility in patients with celiac disease: a meta-analysis of observational studies. Arquivos de gastroenterologia, 2014;51(2):144-150.

11. LEBWOHL B, RUBIO-TAPIA A. Epidemiology, Presentation, and Diagnosis of Celiac Disease. Gastroenteroly , 2020; 160:6375.

12. LEONARD MM, et al. Celiac Disease and Nonceliac Gluten Sensitivity A Review. JAMA, 2017;318(7):647-656.

13. LESLIE LA, et al. Incidence of lymphoproliferative disorders in patients with celiac disease. American journal of hematology, 2012; 87:754-759.

14. LIMA CSA, et al. (Re)significando a doença celíaca na infância e (re)conhecendo as abordagens gerais através de uma revisão narrativa. Revista Eletrônica Acervo Saúde, 2021; 13(5):1-7.

15. MAISIAK E, et al. The impact of symptoms on quality of life before and after diagnosis of coeliac disease: the results from a Polish population survey and comparison with the results from the United Kingdom. BMC Gastroenterology, 2021;21(1):99.

16. MURPHY AK, et al. Celiac Disease in an Adult Presenting as Behavioral Disturbances. The American Journal of Case Reports, 2020; 21: e928337.

17. NATIONAL INSTITUTE FOR HEALTH AND CARE EXCELLENCE. Coeliac disease: recognition, assessment and management. 2015. Deponível em: https://www.nice.org.uk/guidance/ng20/chapter/Recommendations\#monitoring-inpeople-with-coeliac-disease. Acessado em: 8 de junho de 2021.

18. PENNY HA, et al. Non-Responsive Coeliac Disease: A Comprehensive Review from the NHS England National Centre for Refractory Coeliac Disease. Nutrients, 2020; $12(1): 216$.

19. REJ A, SANDERS DS. An update on coeliac disease from the NHS England National Centre for Refractory Coeliac Disease. Clinical medicine, $2021 ; 21(2): 127-130$.

20. RODRIGO L. Doença celíaca: um problema de saúde comum não reconhecido com um diagnóstico muito tardio. Medicina, 2019; 56(1):9.

21. ROSTAMI K, et al. Gluten-free diet indications, safety, quality, labels, and challenges. Nutrients. 2017; 9(8):846.

22. SANTOS AS, RIBEIRO CG. Percepções de doentes celíacos sobre as consequências clínicas e sociais de um possível diagnóstico tardio na doença celíaca. Demetra, 2019; 1-17.

23. SINGH P, et al. Celiac Disease in Women with Infertility. Journal of clinical gastroenterology, 2016; 50(1):33-39.

24. SOOD A, et al. Adult celiac disease: delayed onset or delayed diagnosis? Annals of gastroenterology, 2014; 27, 284-290.

25. TAN IL, et al. Non-classical clinical presentation at diagnosis by male celiac disease patients of older age. European journal of internal medicine, 2020; 83:28-33.

26. THAWANI SP, et al. Risk of neuropathy among 28,232 patients with biopsy-verified celiac disease. JAMA Neurology, 2015;72(7):806-811.

27. ZINGONE F, et al. Psychological morbidity of celiac disease: a review of the literature. United European gastroenteroly jornal, 2015; 3(2):136-145. 